\title{
Positive global solutions of nonlocal boundary value problems for the nonlinear convection reaction-diffusion equations
}

Tianfu Ma* and Baoqiang Yan

${ }^{\text {"Correspondence: }}$ tianfum@msn.com

School of Mathematical Sciences, Shandong Normal University, Jinan, 250014, China

\section{Springer}

\begin{abstract}
In this paper, the nonlocal boundary value problems for a class of nonlinear functional convection reaction-diffusion equations with the singular reaction function are studied by using the method of upper and lower solutions and monotone iterative technique. Some of sufficient results on the existence and uniqueness of positive global solutions or positive solutions for the boundary value problems are presented, which are a generalization of some recent results in the area.
\end{abstract}

MSC: 35B09; 35K57; 35R10; 35J57; 35K67

Keywords: positive global solution; nonlocal boundary value problems; functional convection reaction-diffusion equation; monotone iterative; upper-lower solutions

\section{Introduction}

Convection reaction-diffusion equations arised from various fields of applied sciences and have received extensive attentions during the past several decades and many topics in the mathematical analysis are well developed and applied to various fields of applied sciences. Much of the developed theory in the earlier years can be found in [1-15] and the references therein. However, most of the main concerns in the literature were the global existence of the solutions, blow-up property of the solutions, the qualitative property of the solutions, asymptotic behavior of global solutions and stability or instability of steady-state solutions. In recent years, some attention on positive solutions has been developed (for examples to see [16-28]). This paper is mainly aimed to study the existence and uniqueness of the positive global solutions or positive solutions for a class of nonlinear nonlocal functional convection reaction-diffusion problems with a singular reaction function which depends on both the $u$ and functional value $K * u$, in which the boundary value problem under consideration is as follows:

$$
\begin{cases}u_{t}-\nabla \cdot(D(x, t) \nabla u)+\mathbf{b}(x, t) \cdot \nabla u=f(x, t, u, K * u) & \text { in } Q, \\ \mathcal{B} u=g(x, t) & \text { on } \partial Q, \\ u(x, 0)=u_{0}(x) & \text { in } \Omega .\end{cases}
$$

Here $Q:=\Omega \times(0, T], \partial Q:=\partial \Omega \times(0, T]$, in which $\Omega$ is a bounded domain in $\mathbb{R}^{n}$ with smooth boundary $\partial \Omega$ and $\nabla \cdot(D(x, t) \nabla)+\mathbf{b}(x, t) \cdot \nabla:=\mathcal{A}$ is a second order uniformly elliptic

(c) The Author(s) 2017. This article is distributed under the terms of the Creative Commons Attribution 4.0 International License (http://creativecommons.org/licenses/by/4.0/), which permits unrestricted use, distribution, and reproduction in any medium, provided you give appropriate credit to the original author(s) and the source, provide a link to the Creative Commons license, and indicate if changes were made. 
operator which the coefficients are assumed to be smooth (say Hölder continuous). The elements $a_{i j}(x, t)$ of uniformly positive definite matrix $D(x, t):=\left(a_{i j}(x, t)\right)$ (also called the diffusion coefficient matrix) are in $C^{1}(\bar{Q})$ and the vector $\mathbf{b}(x, t):=\left(b_{1}(x, t), \ldots, b_{n}(x, t)\right)$ is the convection coefficient in which $b_{i}(x, t) \in C(\bar{Q})(1 \leq i \leq n)$. By the uniform ellipticity of $\mathcal{A}$, there exists a positive constant $a_{0}$ such that

$$
a_{i i}(x, t) \geq a_{0} \quad \text { for all } x \in \bar{Q}(i=1,2, \ldots, n) .
$$

$\mathcal{B}$ is one of the boundary operators

$$
\begin{aligned}
& \mathcal{B} u=u \quad \text { on } \partial Q, \\
& \mathcal{B} u=\alpha u_{v}+\beta u, \quad \text { on } \partial Q,
\end{aligned}
$$

where $u_{v}$ denotes the outward normal derivative of $u$ on $\Omega, \alpha:=\alpha(x, t), \beta:=\beta(x, t)$ are both bounded nonnegative function everywhere on the boundary $\partial Q, g:=g(x, t)$ is a nonnegative function and the reaction function $f(x, t, u, v)$ is, in general, a nonlinear function of $(u, v)$. The functional value $K * u$ is given by

$$
K * u:=\int_{\Omega} k(x) u(x, t) d x .
$$

The initial function $u_{0}(x)$ is smooth, nonnegative and satisfies the compatibility condition $u_{0}(x)=0$ on $\partial \Omega$. In addition, we impose the following main hypothesis on the function $k(x)$ and the function $f(x, t, u, v):=f(x, t, u, K * u)$.

Hypothesis (H) (i) The function $k(x)$ is continuous nonnegative on $\bar{\Omega}$ and possesses the following property:

$$
k_{0}=\int_{\Omega} k(x) d x \leq 1
$$

(ii) $f(x, t, 0,0) \geq 0$ and there exists a constant $m_{0}>0$ such that $f(x, t, u, v)$ is a $C^{1}$-function in $(u, v)$ and $f_{v}(x, t, u, v) \geq 0$ for $u, v \in\left[0, m_{0}\right)$.

As in many other cases the existence or nonexistence of positive solutions for (1.1) is closely related to the existence or nonexistence of positive solutions of the corresponding the steady-state problems, so that we consider first the following nonlinear elliptic boundary value problem:

$$
\begin{cases}-\mathcal{A} u=f(x, u, K * u) & \text { in } \Omega, \\ \mathcal{B} u=g(x) & \text { on } \partial \Omega .\end{cases}
$$

Clearly, it is well known that if $f=f(u, K * u)$ is independent of $K * u$ and $u_{0}(x)=0$. Then by the condition (ii) of Hypothesis $(\mathcal{H})$ there exist a parameter $p>0$ and a domain $\Omega_{p}$ such that the problem

$$
\begin{cases}-\mathcal{E} u=f(u, K * u) & \text { in } \Omega_{p}, \\ u(x)=0 & \text { on } \partial \Omega_{p},\end{cases}
$$


(here $\mathcal{E} u:=\mathcal{A} u-\sum_{i=1}^{n} b_{i} u_{x_{i}}$ ) has a positive solution (cf. [21]). Furthermore, if $f(0,0)>0$ and $\lim _{u \rightarrow m_{0}} f(u, v)=\infty$, then a unique global solution $u_{p}$ of the following problem:

$$
\begin{cases}u_{t}-\mathcal{E} u=f(u, K * u) & \text { in } \Omega_{p} \times \mathbb{R}^{+}, \\ u(x, t)=0, & \text { on } \partial \Omega_{p} \times \mathbb{R}^{+}, \\ u(x, 0)=u_{0}(x) & \text { in } \Omega_{p},\end{cases}
$$

exists and converges to a positive solution of (1.4) for a certain domain $\Omega_{p^{\prime}} \subset \Omega_{p}$ (cf. [13$15])$. Here $\Omega_{p}$ is a family of smooth bounded domains in $\mathbb{R}^{n}$ with $p$ as the parameter such that

$$
\bar{\Omega}_{p} \subset \Omega_{q} \quad(p<q), \quad \bigcup_{\alpha} \Omega_{p}=\mathbb{R}^{n}, \quad \text { and } \quad \operatorname{dia}\left(\Omega_{p}\right) \rightarrow 0 \quad(p \rightarrow 0),
$$

where $\operatorname{dia}\left(\Omega_{p}\right)$ denotes the diameter of the domain $\Omega_{p}$.

The purpose of this study is to establish the existence and uniqueness of the positive global solutions or positive solutions for problems (1.1) or problem (1.3). This paper is organized as follows. In Section 2, the discussion focuses on the positive solutions of nonlocal nonlinear functional elliptic boundary value problems (1.3), we first present the maximal and minimal solutions and $C^{2+\alpha}$ nonnegative solutions by monotone iterative technique and Schauder estimates; lastly, some results on a positive local solution and the uniqueness of positive solutions for problem (1.3) are derived. In Section 3, the discussion focuses on the positive global solutions for nonlocal nonlinear convection reactiondiffusion boundary value problems (1.1), we present some results on the unique fixed solution, a strong solution for problem (1.1) by the means of Collatz monotone operator, and we show that every smooth upper solution of the elliptic problem (3.4) gives rise to a nonincreasing solution of the nonlocal convection reaction-diffusion problem (3.5) and $u_{t} \leq 0$ in $\Omega$ provided Hypothesis $(\mathcal{H}$ ) holds; lastly, the sufficient and necessary conditions of positive global solutions and the uniqueness of positive global solutions for problem (1.1) are both given.

\section{Positive solutions of nonlocal nonlinear functional elliptic boundary value problems}

It is well known that various assumptions in the previous literature have been made on the reaction term $f(x, t, u, K * u)$ (we have $K * u=0$, $t$, or $(x, t))$ such as monotonicity, positivity, convexity, concavity, or boundedness, etc., but these assumptions can be relaxed considerably (if not altogether) by using the iteration scheme $(c f .[10,24,26])$. One of the contributions in this paper, of course, in this section will be to emphasize the importance of the applications of upper and lower solutions $(c f .[15,16,21,24])$, which are defined by the following.

Definition 2.1 A function $\breve{u}$ in $C^{2}(\Omega) \cap C(\bar{\Omega})$ is called an upper solution of (1.3) if $\breve{u}$ satisfies the following inequalities:

$$
\begin{cases}-\mathcal{A} \breve{u} \geq f(x, \breve{u}, K * \breve{u}) & \text { in } \Omega, \\ \mathcal{B} \breve{u} \geq g & \text { on } \partial \Omega .\end{cases}
$$


Similarly, $\hat{u}$ in $C^{2}(\Omega) \cap C(\bar{\Omega})$ is called a lower solution of (1.3) if it satisfies the inequalities (2.1) in reversed order. The pair $\hat{u}, \breve{u}$ are said to be ordered if $\hat{u} \leq \breve{u}$ on $\bar{\Omega}$.

Now we suppose that there exist a pair of ordered upper and lower solutions $\breve{u}, \hat{u}$ to (1.3) and define

$$
\begin{aligned}
& \langle\hat{u}, \breve{u}\rangle:=\{u \in C(\bar{\Omega}) ; \hat{u} \leq u \leq \breve{u}\}, \\
& \gamma: \geq \max \left\{-f_{u}(x, t, u, v)-f_{v}(x, t, u, v) k_{0} ; u, v \in\langle\hat{u}, \breve{u}\rangle\right\} .
\end{aligned}
$$

By using either $u^{(0)}=\check{u}$ or $u^{(0)}=\hat{u}$ as the initial iteration we can construct a sequence $\left\{u^{(k)}\right\}$ from the following linear iteration process:

$$
\begin{cases}-(\mathcal{A}-\gamma) u^{(k)}=f\left(x, u^{(k-1)}, K * u^{(k-1)}\right)+\gamma u^{(k-1)} & \text { in } \Omega \\ \mathcal{B} u^{(k)}=g & \text { on } \partial \Omega .\end{cases}
$$

Then we have an existence theorem of the maximal and minimal solutions first as follows.

Theorem 2.1 Let Hypothesis $(\mathcal{H})$ hold, and let $\breve{u}, \hat{u}$ be a pair of ordered upper and lower solutions of (1.3). If $f(x, u, K * u)$ is a smooth function on $\min \hat{u} \leq u \leq \max \breve{u}$. Then there exist two nonnegative solutions $\bar{u}$ and $\underline{u}$ of the problem (1.3) such that $\hat{u} \leq \underline{u} \leq \bar{u} \leq \breve{u}$.

Proof It is clear that $\hat{u}=0$ is a lower solution of (1.3) for domain $\Omega$ by Hypothesis $(\mathcal{H})$. We can assume $f_{u}(x, u, K * u)$ is bounded below for $x \in \Omega$ and $\min \hat{u} \leq u \leq \max \check{u}$, so that $f_{u}(x, u, v)+f_{v}(x, u, v) k_{0}+\gamma>0$ for all $x \in \Omega, u$ in that interval and for given $\gamma$. Now we define the mapping $T$ as follows: $w=T u$ if

$$
\begin{cases}-(\mathcal{A}-\gamma) w=f(x, u, K * u)+\gamma u & \text { in } \Omega \\ \mathcal{B} w=g & \text { on } \partial \Omega\end{cases}
$$

$T$ is completely continuous, since it takes space $C^{\alpha}$ into $C^{2+\alpha}$ by the Schauder estimates for elliptic equations. Furthermore, it is monotone in the sense of Collatz [9], i.e., $u_{1} \leq u_{2}$ implies $T u_{1}<T u_{2}$, provided that $u_{1}$ and $u_{2}$ are restricted to the set $\min \hat{u} \leq u_{1}, u_{2} \leq \max \check{u}$. In fact, if $u_{1} \leq u_{2}$ then

$$
\begin{cases}-(\mathcal{A}-\gamma)\left(T u_{2}-T u_{1}\right)=f\left(x, u_{2}, K * u_{2}\right)-f\left(x, u_{1}, K * u_{1}\right)+\gamma\left(u_{2}-u_{1}\right) & \text { in } \Omega \\ \mathcal{B}\left(T u_{2}-T u_{1}\right)=0 & \text { on } \partial \Omega\end{cases}
$$

Define $F(x, u, v)=f(x, u, v)+\gamma u$. Then $F_{u}(x, u, v)=f_{u}(x, u, v)+f_{v}(x, u, v) k_{0}+\gamma>0$. This implies that $F(x, u, K * u)$ is strictly increasing on $u$, so

$$
\begin{cases}-(\mathcal{A}-\gamma)\left(T u_{2}-T u_{1}\right) \geq 0 & \text { in } \Omega \\ \mathcal{B}\left(T u_{2}-T u_{1}\right)=0 & \text { on } \partial \Omega\end{cases}
$$

Therefore, $T u_{1}<T u_{2}$ in $\Omega$ by the strong maximum principle for elliptic operators. 
Now let $u^{(0)}=\check{u}$ or $u^{(0)}=\hat{u}$ be as the initial iteration and construct a sequence $\left\{u^{(k)}\right\}:=$ $\left\{T u^{(k-1)}\right\}$ from the following linear iteration process:

$$
\begin{cases}-(\mathcal{A}-\gamma) u^{(k)}=f\left(x, u^{(k-1)}, K * u^{(k-1)}\right)+\gamma u^{(k-1)} & \text { in } \Omega, \\ \mathcal{B} u^{(k)}=g & \text { on } \partial \Omega .\end{cases}
$$

Denoting the sequence by $\left\{\bar{u}^{(k)}\right\}$ when $u^{(0)}=\breve{u}$ and by $\left\{\underline{u}^{(k)}\right\}$ when $u^{(0)}=\hat{u}$. Then the sequence $\left\{\bar{u}^{(k)}\right\}$ converges monotonically from above to a maximal solution $\bar{u}_{\max }$ and $\left\{\underline{u}^{(k)}\right\}$ converges monotonically from below to a minimal solution $\underline{u}_{\min }$ by the continuity of $T$ (cf. [16]). Thus $\bar{u}:=\bar{u}_{\max }$ and $\underline{u}:=\underline{u}_{\min }$ are two fixed points of $T$, and furthermore, they are of class $C^{2+\alpha}$ if $f$ satisfies Hypothesis $(\mathcal{H})$ for $0<\alpha<1$. This proves Theorem 2.1.

Corollary 2.1 If solutions $\left\{\bar{u}_{\max }\right\}$ and $\left\{\underline{u}_{\min }\right\}$ are constructed in the proof of Theorem 2.1. Then, for any solution $w$ of the problem (1.3), which satisfies $\hat{u} \leq w \leq \breve{u}$, we have $\underline{u}_{\min } \leq w \leq$ $\bar{u}_{\max }$.

Proof In view of the proof of Theorem 2.1, we have $w=T w, \bar{u}_{1}=T \check{u}$; since $w \leq \breve{u}, T w<T \breve{u}$, or $w<\bar{u}_{1}$. By induction, $w \leq \bar{u}^{(k)}$ for all $k$, hence $w \leq \bar{u}_{\max }$. Similarly, $w \geq \underline{u}_{\min }$, so $\underline{u}_{\min } \leq$ $w \leq \bar{u}_{\max }$.

Hypothesis $(\mathcal{H})$ implies that $\hat{u}=0$ is a lower solution of (1.3) for domain $\Omega$. In order to find a positive solution, we thus only to find a positive upper solution. To do this, we have a result which is similar to [21] as follows.

Theorem 2.2 Let Hypothesis $(\mathcal{H})$ hold. Then the problem (1.3) has at least one positive local solution $u^{+}(x)$.

Proof Following the idea of the proof of Lemma 2.1 in [21] (it is noticed that there $L u=$ $\mathcal{A} u-\sum_{i=1}^{n} b_{i} u_{x_{i}}$ ), we may find a small smooth bounded domain $\Omega^{\prime} \subset \Omega$ such that $d=$ $\operatorname{dia}\left(\Omega^{\prime}\right)$ satisfies the following inequality:

$$
d\left|\frac{\partial a_{i i}}{\partial x_{i}}+b_{1}\right| \leq a_{i i}-\frac{a_{0}}{2}, \quad x \in \overline{\Omega^{\prime}}, i=1,2, \ldots, n
$$

where $a_{0}>0$ is a constant that appeared in (1.2). Without any loss of generality we may assume that $x^{\prime}=(0,0, \ldots, 0)$ and $x^{\prime \prime}=(d, 0, \ldots, 0)$ are the two boundary points of $\overline{\Omega^{\prime}}$ along the $x_{1}$-axis. Let $M$ be any constant satisfying $M \geq(f(x, 0,0)+\gamma) / a_{0}$, and let $\breve{u}(x):=M\left(d^{2}-\right.$ $x_{1}^{2}$ ). Then $\check{u} \geq 0$ on $\overline{\Omega^{\prime}}$ and

$$
\begin{aligned}
-\mathcal{A} \check{u} & =M\left[2 a_{11}+2 x_{1}\left(\frac{\partial a_{11}}{\partial x_{1}}+b_{1}\right)\right] \\
& \geq 2 M\left(a_{11}-d\left|\frac{\partial a_{11}}{\partial x_{1}}+b_{1}\right|\right) \\
& \geq M a_{0} \\
& \geq f(x, 0,0)+\gamma .
\end{aligned}
$$


Since $\breve{u} \leq M d^{2}$ and $K * \check{u} \leq M k_{0} d^{2}$ there exists a constant $\delta>0$ such that

$$
\begin{aligned}
f(x, \check{u}, K * \check{u}) & =f_{U}(x, \xi, \eta) U+f_{V}(x, \xi, \eta) V+f(x, 0,0) \\
& \leq f(x, 0,0)+\gamma \quad \text { as } d \leq \delta
\end{aligned}
$$

where $U:=\breve{u}, V:=K * \check{u}, \xi:=\xi(x)$ and $\eta:=\eta(x)$ are some intermediate values between $\check{u}$ and 0 and between $(K * \breve{u})$ and 0 , respectively. This proves that, for some small $d, \breve{u}(x)=$ $M\left(d^{2}-x_{1}^{2}\right)$ is a positive upper solution of (1.3). Combining with the fact that $\hat{u}:=u=0$ is a lower solution of (1.3), it follows from Theorem 2.1 that there exists at least one positive local solution $u^{+}(x)$ of the problem (1.3).

As is well known, the monotone iterative scheme for elliptic boundary value problems is based on a positivity lemma which plays a fundamental role in nonlinear elliptic boundary value problems. A lemma ( $c f$. [15]) under consideration is introduced here for the sake of discussing the uniqueness of the positive solutions.

Lemma 2.1 Let c, $\alpha, \beta$ be bounded nonnegative functions which are not both identically zero, and let $w \in C^{2}(\Omega)$ satisfy the following inequalities:

$$
\begin{cases}-\mathcal{A} w+c w \geq 0 & \text { in } \Omega \\ \mathcal{B} w \geq 0 & \text { on } \partial \Omega\end{cases}
$$

Then $w \geq 0$ in $\bar{\Omega}$. Moreover, $w>0$ in $\Omega$ unless $w \equiv 0$.

Now if $u_{1}, u_{2} \in\langle\hat{u}, \breve{u}\rangle$, there exist two bounded nonnegative functions $c_{1}(x), c_{2}(x)$ in $\Omega$ such that function $f(x, u, K * u)$ satisfies the following inequality:

$$
f\left(x, u_{1}, K * u_{1}\right)-f\left(x, u_{2}, K * u_{2}\right) \geq-c_{1}(x)\left(u_{1}-u_{2}\right)-c_{2} K *\left(u_{1}-u_{2}\right) \quad \text { in } \Omega .
$$

Then we have the following uniqueness result of positive solutions for problem (1.3).

Theorem 2.3 Let $\beta$ be a function which not identically zero, and let $\breve{u}(x), \hat{u}(x)$ be a pair of ordered nonnegative upper and lower solutions of $(1.3)$. If the function $f(x, u, K * u)$ satisfies (2.4), then the positive solution of the problem (1.3) in $\langle\hat{u}, \breve{u}\rangle$ is unique.

Proof It is clear that positive solutions exist from Theorem 2.1. Let $u_{1}, u_{2} \in\langle\hat{u}, \breve{u}\rangle$ be two positive solutions with $u_{1} \leq u_{2}$. Suppose $w=u_{1}-u_{2}$, then $w \leq 0$ and by $(2.4)$

$$
\begin{cases}-\mathcal{A} w=f\left(x, u_{1}, K * u_{1}\right)-f\left(x, u_{2}, K * u_{2}\right) \geq 0 & \text { in } \Omega \\ \mathcal{B} w=g(x)-g(x)=0 & \text { on } \partial \Omega\end{cases}
$$

Applying Lemma 2.1 we then have $u_{1}=u_{2}$ in $\bar{\Omega}$. The uniqueness of the positive solutions is proved. 


\section{Positive global solutions of nonlocal functional reaction-diffusion boundary value problems}

In this section we go back to the problem (1.1) and devote ourselves to a discussion of the existence and uniqueness of the positive global solutions or positive solutions. The boundary operator $\mathcal{B} u$ is one of the operators

$$
\begin{aligned}
& \mathcal{B} u(x, t)=u(x, t) \quad \text { on } \partial Q, \\
& \mathcal{B} u(x, t)=\alpha u_{v}(x, t)+\beta u(x, t) \quad \text { on } \partial Q, \quad \text { and } \\
& u(x, 0)=u_{0}(x) \quad \text { in } \Omega .
\end{aligned}
$$

Now we hereafter use $\mathcal{L} u=u_{t}-\mathcal{A} u$ and recall the definition of a pair of ordered upper and lower solutions on problem (1.1) first as follows.

Definition 3.1 For every finite $T$, a function $\breve{u}(x, t) \in C(\bar{Q}) \cap C^{1,2}(Q)$ is called an upper solution of (1.1) if $\breve{u}$ satisfies the following inequalities:

$$
\begin{cases}\mathcal{L} \breve{u} \geq f(x, t, \breve{u}, K * \breve{u}) & \text { in } Q, \\ \mathcal{B} \breve{u} \geq g(x, t) & \text { on } \partial Q, \\ u(x, 0) \geq u_{0}(x) & \text { in } \Omega .\end{cases}
$$

A lower solution $\hat{u}(x, t) \in C(\bar{Q}) \cap C^{1,2}(Q)$ can be defined by reversing the inequalities in (3.2), and the pair $\hat{u}, \check{u}$ are said to be ordered if $\hat{u} \leq \breve{u}$ on $\bar{Q}$. The set of functions $u \in C(\bar{Q})$ such that $\hat{u} \leq u \leq \breve{u}$ in $\bar{Q}$ is again denoted by $\langle\hat{u}, \breve{u}\rangle$.

Clearly, every solution of (1.1) is an upper solution as well as a lower solution. Given a pair of upper and lower solutions $\breve{u}(x, t), \hat{u}(x, t)$, we choose $\gamma$ as in (2.2) such that $f_{u}(x, t, u, v)+$ $f_{v}(x, t, u, v) k_{0}+\gamma>0$ on the sector $\min \hat{u}(x, t) \leq u, v \leq \max \check{u}(x, t)$. Defining $\bar{u}^{(1)}$ by

$$
\begin{cases}\mathcal{L} \bar{u}^{(1)}+\gamma \bar{u}^{(1)}=f(x, t, \check{u}, K * \check{u})+\gamma \check{u} & \text { in } Q, \\ \mathcal{B} \bar{u}^{(1)}=g & \text { on } \partial Q, \\ u^{(1)}(x, 0)=u_{0}(x) & \text { in } \Omega .\end{cases}
$$

By the maximum principle for a parabolic equation it is easily seen that $\bar{u}^{(1)}(x, t)<\check{u}(x, t)$ in $\Omega$. The mapping $\check{u}(x, t) \rightarrow \bar{u}^{(1)}(x, t)$ is denoted by $\bar{u}^{(1)}=\mathcal{J} \breve{u}$. $\mathcal{J}$ again is a monotone operator in the sense of Collatz, and similarly doing $\underline{u}^{(1)}=\mathcal{J} \hat{u}$, by using the monotone arguments go through exactly as before ( $c f$. [12]), then we can obtain the following theorem.

Theorem 3.1 Let Hypothesis $(\mathcal{H})$ hold, and let $\breve{u}(x, t), \hat{u}(x, t)$ in $\bar{Q}$ be a pair of upper and lower solutions. Defining sequences $\left\{\bar{u}^{(m)}\right\}$ and $\left\{\underline{u}^{(m)}\right\}$ by $\bar{u}^{(m)}:=\mathcal{J} \bar{u}^{(m-1)}$ and $\underline{u}^{(m)}:=\mathcal{J} \underline{u}^{(m-1)}$, respectively, in which $\bar{u}^{(1)}:=\mathcal{J} \check{u}$ and $\underline{u}^{(1)}:=\mathcal{J} \hat{u}$. If there exists $\gamma$ such that

$$
f_{u}(x, t, u, v)+f_{v}(x, t, u, v) k_{0}+\gamma>0 \quad \text { in } \min _{\Omega} \hat{u}<u, v<\max _{\Omega} \check{u},
$$


then the sequences $\left\{\bar{u}^{(m)}\right\}$ and $\left\{\underline{u}^{(m)}\right\}$ are monotone decreasing and increasing, respectively, and a unique fixed solution $u$ satisfying

$$
\lim _{m \rightarrow \infty} \bar{u}^{(m)}=\mathcal{J} u=u=\mathcal{J} u=\lim _{m \rightarrow \infty} \bar{u}^{(m)}
$$

is a strong solution of problem (1.1).

The following corollary is immediate from Theorem 3.1, if $g$ is time independent.

Corollary 3.1 Let Hypothesis $(\mathcal{H})$ hold, and let $\bar{u}(x)$ and $\underline{u}(x)$ be a pair of upper and lower solutions of the following elliptic boundary value problem:

$$
\begin{cases}-\mathcal{A} u=f(x, u, K * u) & \text { in } \Omega, \\ \mathcal{B} u=g & \text { on } \partial \Omega .\end{cases}
$$

Then, for any solution $u(x) \in\langle\underline{u}, \bar{u}\rangle$, we can obtain a global regular solution $u(x, t)$ which satisfies $\underline{u}(x) \leq u(x, t) \leq \bar{u}(x)$ for all $t>0$.

Now if $u(x)$ is an upper solution of the elliptic problem (1.3), then as we have seen, it can be made the starting point of a monotone decreasing sequence of iterates and we may obtain the corresponding construction solution $u(x, t)$ which is monotone decreasing on time $t$. Thus we have the following result.

Theorem 3.2 Let Hypothesis $(\mathcal{H})$ hold, and let $\bar{u}(x)$ be an upper solution of the following problem:

$$
\begin{cases}-\mathcal{A} u=f(x, u, K * u) & \text { in } Q, \\ u=0 & \text { on } \partial \Omega .\end{cases}
$$

If $u(x, t)$ is a solution of the following problem:

$$
\begin{cases}\mathcal{L} u=f(x, u, K * u) & \text { in } Q \\ u=0 & \text { on } \partial Q \\ u(x, 0)=\bar{u}(x) & \text { in } \Omega .\end{cases}
$$

Then $u_{t} \leq 0$ in $Q$, i.e., $u(x, t)$ is nonincreasing on $t$.

Proof Defining a sequence of functions $\left\{u^{(n)}\right\}$ in $Q$ by $u^{(0)}(x, t)=\bar{u}(x):=\bar{u}$, and for $n \geq 1$

$$
\begin{cases}\mathcal{L} u^{(n)}+\gamma u^{(n)}=f\left(x, u^{(n-1)}, K * u^{(n-1)}\right)+\gamma u^{(n-1)} & \text { in } Q, \\ u^{(n)}=0 & \text { on } \partial Q \\ u^{(n)}(x, 0)=\bar{u}(x), & \text { in } \Omega .\end{cases}
$$

Then the function sequence $\left\{u^{(n)}(x, t)\right\}$ is nondecreasing and

$$
\bar{u}(x) \geq u^{(1)}(x, t) \geq \cdots \geq u^{(n-1)}(x, t) \geq u^{(n)}(x, t) \geq \cdots .
$$


In fact, we first have

$$
\left\{\begin{array}{l}
\mathcal{L}\left(u^{(1)}-\bar{u}\right)+\gamma\left(u^{(1)}-\bar{u}\right)=-[f(x, \bar{u}, K * \bar{u})-\mathcal{A} \bar{u}] \geq 0 \\
\mathcal{B}\left(u^{(1)}-\bar{u}\right)=g(x, t)-\mathcal{B} \bar{u} \leq 0
\end{array}\right.
$$

This gives $\bar{u} \geq u^{(1)}$ by the strong maximum principle. Furthermore, we can easily prove $u^{(n-1)}(x, t) \geq u^{(n)}(x, t)$ by induction for $n \in \mathbb{N}$, the inequality (3.7) comes into existence. Suppose $u^{(n)}(x, t) \rightarrow v(x, t)(n \rightarrow \infty)$, then the limit function $v(x, t)$ must be a solution of the following problem:

$$
\begin{cases}\mathcal{L} v=f(x, v, K * v) & \text { in } Q \\ v=0 & \text { on } \partial Q \\ v(x, 0)=\bar{u}(x), & \text { in } \Omega .\end{cases}
$$

Thus, by uniqueness, $v(x, t)=u(x, t)$ in $Q$. Now we find by differentiating (3.6) with respect to $t$,

$$
\begin{cases}\mathcal{L}\left(u^{(n)}\right)_{t}+\gamma\left(u^{(n)}\right)_{t}=f_{U}(x, U, V) U_{t}+f_{V}(x, U, V) V_{t} & \text { in } Q \\ \left(u^{(n)}\right)_{t}=0 & \text { on } \partial Q\end{cases}
$$

where $U:=u^{(n-1)}, V:=K * u^{(n-1)}$. Clearly, the right hand side of the first equality above is a bounded function in $Q$. Define, if $\delta>0$,

$$
w_{n}=\frac{u^{(n)}(x, \delta)-u^{(n)}(x, 0)}{\delta}, \quad x \in \Omega
$$

then $w_{n} \leq 0$ from (3.6) and (3.7), hence $\left(u^{(n)}(x, 0)\right)_{t} \leq 0, x \in \Omega$. Therefore $\left(u^{(n)}\right)_{t} \leq 0$ $(x \in \Omega)$ by the strong maximum principle for parabolic equations. Similar to the proof of Theorem 2.1, we can show that $u^{(n)}(x, t)$ tends to $u(x, t)$ in $C^{1+\alpha}$ on $t$ in $Q$, thus $u_{t}(x, t) \leq 0$ in $Q$. The proof is completed.

Remark 3.1 Theorem 3.2 illustrates that every smooth upper solution $\bar{u}(x)$ of the elliptic problem (3.4) gives rise to a nonincreasing solution $u(x, t)$ of the convection reactiondiffusion problem (3.5), and $u_{t} \leq 0$ in $\Omega$ provided Hypothesis $(\mathcal{H})$ holds.

It is well known that the maximum principle of parabolic or elliptic boundary value problems in the method of upper and lower solutions of convection reaction-diffusion boundary value problems plays a fundamental role, especially in the construction of monotone sequences. This role is reflected in Lemma 3.1 which is called the positive lemma (see [15]), for the time-dependent and the steady-state problem, respectively.

Lemma 3.1 Let $w \in C(\bar{Q}) \cap C^{1,2}(Q)$ be such that

$$
\begin{cases}\mathcal{L} w+c w \geq 0 & \text { in } Q \\ \mathcal{B} w \geq 0 & \text { on } \partial Q \\ w(x, 0) \geq 0 & \text { in } \Omega\end{cases}
$$


where $\alpha, \beta \geq 0, \alpha+\beta>0$ on $\partial Q$, and $c:=c(x, t)$ is a bounded function in $Q$. Then $w(x, t) \geq 0$ in $Q$. Moreover, $w(x, t)>0$ in $Q$ unless $w(x, t) \equiv 0$.

In many convection reaction-diffusion boundary value problems as (1.1), if the reaction term $f(x, t, u, K * u)$ is a $C^{1}$-function on $u$ and $K * u$, and if the following data possesses the nonnegative property:

$$
f(x, t, 0,0) \geq 0, \quad g(x, t) \geq 0, \quad u_{0}(x) \geq 0,
$$

then combining with the fact every solution of the problem (1.1) is an upper solution as well as a lower solution, as a result the existence of a bounded global solution in $\bar{\Omega} \times \mathbb{R}^{+}$ follows (cf. [15]).

Theorem 3.3 If there exist two positive constants $c_{1}, c_{2}$ with $c_{1}<c_{2}$ such that $f(x, t, u, K * u)$ is a $C^{1}$-function on $u, K * u \in\left[c_{1}, c_{2}\right]$, and

$$
\begin{aligned}
& f\left(x, t, c_{1}, K * c_{1}\right) \geq 0, \quad f\left(x, t, c_{2}, K * c_{2}\right) \leq 0 \quad \text { in } \Omega \times \mathbb{R}^{+}, \\
& c_{1} \beta(x, t) \leq g(x, t) \leq c_{2} \beta(x, t) \quad \text { on } \partial \Omega \times \mathbb{R}^{+} .
\end{aligned}
$$

Then, for any $u_{0} \in\left[c_{1}, c_{2}\right]$, problem (1.1) has a unique bounded global solution $u(x, t)$ in $\Omega \times \mathbb{R}^{+}$such that $u(x, t) \in\left[c_{1}, c_{2}\right]$.

Proof Let $\check{u}=c_{2}, \hat{u}=c_{1}$, then by (3.10)

$$
\begin{cases}\mathcal{L} \check{u}=0 \geq f\left(x, t, c_{2}, K * c_{2}\right)=f(x, t, \check{u}, K * \check{u}) & \text { in } \Omega \times \mathbb{R}^{+}, \\ \mathcal{B} \check{u}=\alpha \check{u}_{v}+\beta \check{u}=c_{2} \beta(x, t) \geq g(x, t) & \text { on } \partial \Omega \times \mathbb{R}^{+}, \\ \check{u}=c_{2} & \text { in } \Omega .\end{cases}
$$

This shows that $\breve{u}=c_{2}$ is an upper solution when $u_{0} \leq c_{2}$. The same reasoning shows that $\hat{u}=c_{1}$ is a lower solution when $u_{0} \geq c_{1}$. The result of the theorem follows from Theorem 3.1.

Remark 3.2 We see, from the proof of Theorem 3.3, that the condition (3.10) shows that the pair $c_{1}, c_{2}$ is a pair of positive upper and lower solutions. So, as a result, Theorem 3.3 may be given in another form as follows.

Corollary 3.2 If there exist $\breve{u}, \hat{u}$ which are a pair of positive upper and lower solutions such that $f(x, t, u, K * \breve{u})$ is a $C^{1}$-function in $u, K * \check{u} \in[\hat{u}, \breve{u}]$ and

$$
\hat{u} \beta(x, t) \leq g(x, t) \leq \check{u} \beta(x, t) \quad \text { on } \partial \Omega \times \mathbb{R}^{+},
$$

then, for any $u_{0} \in\langle\hat{u}, \breve{u}\rangle$, problem (1.1) has a unique bounded global solution $u(x, t)$ in $\Omega \times$ $\mathbb{R}^{+}$such that $u(x, t) \in\langle\hat{u}, \breve{u}\rangle$.

Clearly, in this situation $\hat{u}=0$ is a lower solution of the problem (1.1). An immediate consequence from Theorem 3.3 is the following sufficient and necessary conditions for the existence of positive solutions. 
Theorem 3.4 Let Hypothesis (H) hold, and let condition (3.9) hold and not all the three functions are identically zero. If $f(x, t, u, K * u)$ is a $C^{1}$-function on $u, K * u \in \mathbb{R}^{+}$. Then problem (1.1) has a unique positive solution if and only if there exists a positive upper solution.

We are now in a position to give the uniqueness result of positive global solution for problem (1.1) as follows.

Theorem 3.5 Under Hypothesis $(\mathcal{H})$, let function $f(x, t, u, K * u)$ be a $C^{1}$-function in $u$, $K * u \in \mathbb{R}^{+}$, and let the condition (3.9) hold and not all the three functions are identically zero. If for every finite time $T$ there is a bounded function $M(x, t)$ such that for $u \geq 0$

$$
f_{u}(x, t, u, v) u+f_{v}(x, t, u, v) v \leq M(x, t) u \quad \text { in } Q
$$

then for problem (1.1) there exists a unique positive global solution.

Proof By Hypothesis $(\mathcal{H})$, the mean-value theorem gives

$$
\begin{aligned}
f(x, t, u, K * u) & =f_{u}(x, t, \xi, \eta) u+f_{(K * u)}(x, t, \xi, \eta)(K * u)+f(x, t, 0,0) \\
& \geq f_{u}(x, t, \xi, \eta) u \text { in } Q,
\end{aligned}
$$

where $\xi:=\xi(x, t)$ and $\eta:=\eta(t)$ are some intermediate values between $u$ and 0 and between $t$ and 0 , respectively.

Now if we write $c(x, t):=-f_{u}(x, t, \xi, \eta)$. Then the solution $u$ satisfies the inequalities (3.8), which implies that either $u=0$ or $u>0$ in $Q$. Since $u$ is positive in $Q$, otherwise $u=0$ only if the three functions in (3.9) all are identically zero. Thus $u:=\hat{u}$ may be referred to a positive lower solution of problem (1.1). Suppose that $w$ is a solution of the following problem:

$$
\begin{cases}\mathcal{L} w=M w+f(x, t, 0,0) & \text { in } Q, \\ \mathcal{B} w=g(x, t) & \text { on } \partial Q, \\ w(x, 0)=u_{0}(x) & \text { in } \Omega .\end{cases}
$$

Then $w$ must be a positive upper solution of the problem (1.1). In fact, writing $\check{u}:=w$ and applying the mean-value theorem, $\breve{u}$ satisfies

$$
f(x, t, \breve{u}, K * \breve{u})=f_{\breve{u}}(x, t, \xi, \eta) \check{u}+f_{(K * \breve{u})}(x, t, \xi, \eta)(K * \breve{u})+f(x, t, 0,0),
$$

where $\xi:=\xi(x, t)$ and $\eta:=\eta(t)$ are some intermediate values between $\breve{u}$ and 0 and between $K * \check{u}$ and 0 , respectively. Combining with Hypothesis $(\mathcal{H})$ and the inequality (3.11) we have

$$
f(x, t, \breve{u}, K * \breve{u}) \leq M(x, t) \check{u}+f(x, t, 0,0) \quad \text { in } Q \text {. }
$$


Hence

$$
\begin{cases}\mathcal{L} \breve{u}=M \check{u}+f(x, t, 0,0) \geq f(x, t, \breve{u}, K * \breve{u}) & \text { in } Q, \\ \mathcal{B} \breve{u}=g(x, t) \geq 0 & \text { on } \partial Q, \\ \check{u}(x, 0)=u_{0}(x) \geq 0 & \text { in } \Omega .\end{cases}
$$

That is, $\breve{u}$ is a positive upper solution of the problem (1.1). Therefore, a unique positive global solution is found immediately from Theorem 3.4. This proves the theorem.

Remark 3.3 The condition (3.11) in Theorem 3.5 ensures the existence of a unique positive global solution in $\Omega \times \mathbb{R}^{+}$but is not necessarily uniformly bounded. As for the discussion of bounded positive global solutions of the problem (1.1) will be still a very interesting work.

\section{Competing interests}

The authors declare that there is no conflict of interests regarding the publication of this paper.

\section{Authors' contributions}

Both authors contributed equally in this article. They read and approved the final manuscript.

\section{Acknowledgements}

The authors would like to thank the anonymous reviewers and editors for their valuable comments and suggestions which improve the presentation of the paper.

This work is supported by the NSFC of China (61603226) and the Fund of Science and Technology Plan of Shandong Province (2014GGH201010).

Received: 21 October 2016 Accepted: 30 December 2016 Published online: 10 January 2017

\section{References}

1. Agmon, S, Douglis, A, Nirenberg, L: Estimates near the boundary for solutions of elliptic partial differential equations satisfying general boundary conditions, I. Commun. Pure Appl. Math. 12, 623-727 (1959)

2. Amann, H: Dynamic theory of quasilinear parabolic systems II. Reaction-diffusion systems. Differ. Integral Equ. 3, 13-75 (1990)

3. Amann, H: Existence and stability of solutions for semi-linear parabolic systems, and applications to some diffusion reaction equations. Proc. R. Soc. Edinb. 81, 35-47 (1978)

4. Amann, $\mathrm{H}$ : Nonlinear elliptic equations with nonlinear boundary conditions. In: Proc. of the 2th Scheveningen Conf. on Differential Equations. Mathematics Studies, vol. 21, pp. 43-63. North Holland, Amsterdam (1976)

5. Amann, H, Crandall, M: On some existence theorems for semi-linear elliptic equations. Indiana Univ. Math. J. 27, 779-790 (1978)

6. Sattinger, DH: Monotonic methods in nonlinear elliptic and parabolic boundary value problems. Indiana Univ. Math. J. 21, 979-1000 (1971)

7. Anderson, JR: Local existence and uniqueness of solutions of degenerate parabolic equations. Commun. Partial Differ. Equ. 16, 105-143 (1991)

8. Afrouzi, GA, Naghizadeh, Z, Mahdavi, S: Monotone methods in nonlinear elliptic boundary value problem. Int. J. Nonlinear Sci. 7, 283-289 (2009)

9. Collatz, L: Functional Analysis and Numerical Mathematics. Academic Press Inc., New York and London (1966)

10. Cohen, DS: Multiple stable solutions of nonlinear boundary value problems arising in chemical reactor theory. SIAM J. Appl. Math. 20, 1-13 (1971)

11. Levine, HA, Sacks, PE: Some existence and nonexistence theorems for solutions of degenerate parabolic equations. J. Differ. Equ. 52, 135-161 (1984)

12. Ladyzenskaja, OA, Solonnikov, VA, Uralceva, NN: Linear and Quasi-Linear Equations of Parabolic Type. AMS Trans. Math. Monographs, vol. 23 (1968)

13. Acker, A, Walter, W: On the global existence of solutions of parabolic differential equations with a singular nonlinear term. Nonlinear Anal. 2, 499-504 (1978)

14. Acker, A, Walter, W: The Quenching Problem for Nonlinear Partial Differential Equations. Lecture Notes in Math., vol. 563. Springer, Berlin (1976)

15. Pao, CV: Nonlinear Parabolic and Elliptic Equations. Plenum Press, New York (1992)

16. Amann, $\mathrm{H}$ : On the existence of positive solutions of nonlinear elliptic boundary value problems. Indiana Univ. Math. J. 21, 125-146 (1971)

17. Ma, TF: Remarks on an elliptic equation of Kirchhoff type. Nonlinear Anal. 63, 1967-1977 (2005)

18. Ma, TF: Positive solutions for a nonlocal fourth order equation of Kirchhoff type. Discrete Contin. Dyn. Syst. Supplement 694-703 (2007)

19. Ma, TF, Rivera, JEM: Positive solutions for a nonlinear nonlocal elliptic transmission problem. Appl. Math. Lett. 16, 243-248 (2003) 
20. Alves, CO, Corrêa, FJSA, Ma, TF: Positive solutions for a quasilinear elliptic equation of Kirchhoff type. Comput. Math. Appl. 49, 85-93 (2005)

21. Pao, CV: Quenching problem of functional parabolic equation. Dyn. Contin. Discrete Impuls. Syst. 8, 89-98 (2001)

22. Pao, CV: Positive solutions of a nonlinear boundary-value problem of parabolic type. J. Differ. Equ. 22, 145-163 (1976)

23. Amann, H, Laestch, T: Positive solutions of convex nonlinear eigenvalue problems. Indiana Univ. Math. J. 25, 259-270 (1976)

24. Keller, HB: Elliptic boundary value problems suggested by nonlinear diffusion processes. Archives Rat. Mech. Anal. 35 , 363-381 (1969)

25. Smoller, J: Shock Waves and Reaction-Diffusion Equations. Springer, New York (1994)

26. Keller, HB: Positive solutions of some nonlinear eigenvalue problems. J. Math. Mech. 19, 278-295 (1969)

27. Pao, CV, Ruan, WH: Positive solutions of quasilinear parabolic systems with Dirichlet boundary condition. J. Math. Anal. Appl. 333, 472-499 (2007)

28. Yan, B, Ma, T: The existence and multiplicity of positive solutions for a class of nonlocal elliptic problems. Boundary Value Problems 165 (2016). doi:10.1186/s13661-016-0670-z

\section{Submit your manuscript to a SpringerOpen ${ }^{\ominus}$ journal and benefit from:}

- Convenient online submission

Rigorous peer review

- Immediate publication on acceptance

- Open access: articles freely available online

- High visibility within the field

- Retaining the copyright to your article 